\title{
A rare combination of cardiovascular anomaly: Supravalvular aortic stenosis and congenital absence of right coronary artery
}

\author{
Yunfei Ling ${ }^{1}$, Song Jiayi ${ }^{1}$, Tiange $\mathrm{Li}^{1}$, and $\mathrm{Ke} \mathrm{Lin}^{1}$ \\ ${ }^{1}$ Sichuan University West China Hospital
}

December 23, 2021

\begin{abstract}
We reported a case of a 3-month-old infant presented with supravalvular aortic stenosis with congenital right coronary artery deficiency. According to cardiovascular CT results, Doty technique was adopted to restore the aortic root geometry under cardiopulmonary bypass. An angioplasty was performed to establish right coronary blood flow at the same time. The patient had no abnormal cardiac symptoms after surgery.
\end{abstract}

Title Page

A rare combination of cardiovascular anomaly: Supravalvular aortic stenosis and congenital absence of right coronary artery

Yunfei Ling ${ }^{1}$, Jiayi Song ${ }^{1}$, Tiange $\mathrm{Li}^{1}$, Ke Lin ${ }^{1 \#}$

${ }^{1}$ Department of cardiovascular surgery, West China Hospital, Sichuan University, Chengdu 610041, People's Republic of China

\# Corresponding author: Ke Lin, $\mathrm{MD}, \mathrm{PhD}$

Phone: + 862885422897

Fax: +862885422897

Email: pangzai356249@126.com

A rare combination of cardiovascular anomaly: Supravalvular aortic stenosis and congenital absence of right coronary artery

Yunfei Ling ${ }^{1}$, Jiayi Song ${ }^{1}$, Tiange $\operatorname{Li}^{1}$, Ke Lin ${ }^{1 \#}$

${ }^{1}$ Department of cardiovascular surgery, West China Hospital, Sichuan University, Chengdu 610041, People's Republic of China

\# Corresponding author: Ke Lin, MD, PhD

Phone: + 862885422897

Fax: + 862885422897

Email: pangzai356249@126.com

\section{Abstract}

We reported a case of a 3-month-old infant presented with supravalvular aortic stenosis with congenital right coronary artery deficiency. According to cardiovascular CT results, Doty technique was adopted to restore 
the aortic root geometry under cardiopulmonary bypass. An angioplasty was performed to establish right coronary blood flow at the same time. The patient had no abnormal cardiac symptoms after surgery.

Keywords: Congennital heart disease

A 3-month-old infant was referred for further intervention due to systolic murmurs found at birth. Echocardiography demonstrated a supravalvular aortic stenosis (SVAS) at the sinutubular junction level, bicuspid aortic valves (BAV) with the raphe on the left cusp, mild aortic regurgitation and secundum atrial septal defect (SASD) (Fig. A, B). Computed tomography (CT) and three-dimensional reconstruction suggested the absence of right coronary artery (Fig. C, D). Left coronary was demonstrated features of stenosis at the ostium (Fig. E). The patient then received surgical intervention under cardiopulmonary bypass. Exploration during surgery revealed that main branch of the right coronary artery was present but not connected to the aorta, so an angioplasty was performed to recover right coronary blood flow. The SASD was repaired using autologous pericardium and left coronary artery received patch augmentation. Doty technique was adopted to restore the aortic root geometry. This technique of extended aortoplasty for symmetric reconstruction of the aorta should provide more predictable relief of aortic obstruction and improved function of the aortic valve $^{1}$.

SVAS is characterized by the stricture of the lumen of the ascending aorta, especially in the supravalvular aorta at the sinutubular junction. SVAS may occur as an isolated defect or combined with other angiostenosis, especially stenosis of pulmonary artery, and usually with arrhythmia at the same time ${ }^{2}$. SVAS can be classified into two categories based on the degree of involvement of the ascending aorta described by echocardiogram reports: discrete SVAS and diffuse $\mathrm{SVAS}^{3}$. Discrete type is characterized by a localized ring-like thickening at the sinutubular junction that results in an hourglass-shaped aortic root while diffuse SVAS is characterized by tubular hypoplasia and thickening of the entire ascending aorta ${ }^{4}$.

Some successful cases reported angioplasty of coronary artery performing SVAS repair at the same time, but these studies primarily described cases in the left coronary artery. Congenital absence of RCA is probably related to dysplasia or congenital occlusion of the RCA during the development of embryo. This kind of may lead to the deficiency of blood supply in sinoatrial and atrioventricular node, eventually causing their dysfunction. Until now, there are few reports on congenital absence of RCA. This may because RCA has non-specific clinical features. We adopted angioplasty to establish right coronary blood flow for the patient. Angioplasty can improve blood supply of the heart without increasing the risk of major complications.

This case image also suggested that compared to echocardiography or catheter angiography, cardiovascular CT can provide excellent visualization of complex congenital anatomies. Anyway, we treated this case with Doty technique and angioplasty procedures mentioned above. Short-term prognosis of the patient revealed good.

\section{CONFLICT OF INTERESTS}

The authors declare that there are no conflict of interests.

\section{ETHICS STATEMENT}

This manuscript and all of its content meet the ethical guidelines, including adherence to the legal requirements of the study country. The need for patient consent was waived.

\section{FUNDING}

This study was funded by 1.3.5 project for disciplines of excellence-Clinical Research Incubation Project, West China Hospital, Sichuan University (ZYJC21064).

Figure 1 (A) Echocardiography of the aorta revealed the stenosis at the sinutubular junction level with a diameter of $4.7 \mathrm{~mm}$ and BAV with mild aortic regurgitation. (B) Echocardiography on the atrium level suggested SASD and mild pulmonary arterial hypertension. (C, D and F) CT and three-dimensional reconstruction confirmed the significant SVAS and demonstrated the absence of right coronary artery. (E) Left 
coronary origin was above the level of Valsalva sinus and demonstrated features of stenosis at the ostium. $(\mathrm{G}$ and $\mathrm{H})$ Exploration during surgery revealed that main branch of the right coronary artery was present but not connected to the aorta.

\section{Reference}

1. Doty DB, Polansky DB, Jenson CB: Supravalvular aortic stenosis. Repair by extended aortoplasty. J Thorac Cardiovasc Surg 74:362-371, 1977.

2. Mirko, Baglivo, Sarah, et al: Atrial septal defects, supravalvular aortic stenosis and syndromes predisposing to aneurysm of large vessels. Acta bio-medica : Atenei Parmensis 90:53-57.

3. Ibarra C, Spigel Z, Msn R, et al: Surgical Techniques in Management of Supravalvular Aortic Stenosis in Children. The Annals of Thoracic Surgery, 2020.

4. Mitchell MB, Goldberg SP: Supravalvar aortic stenosis in infancy. Semin Thorac Cardiovasc Surg Pediatr Card Surg Annu 14:85-91, 2011.

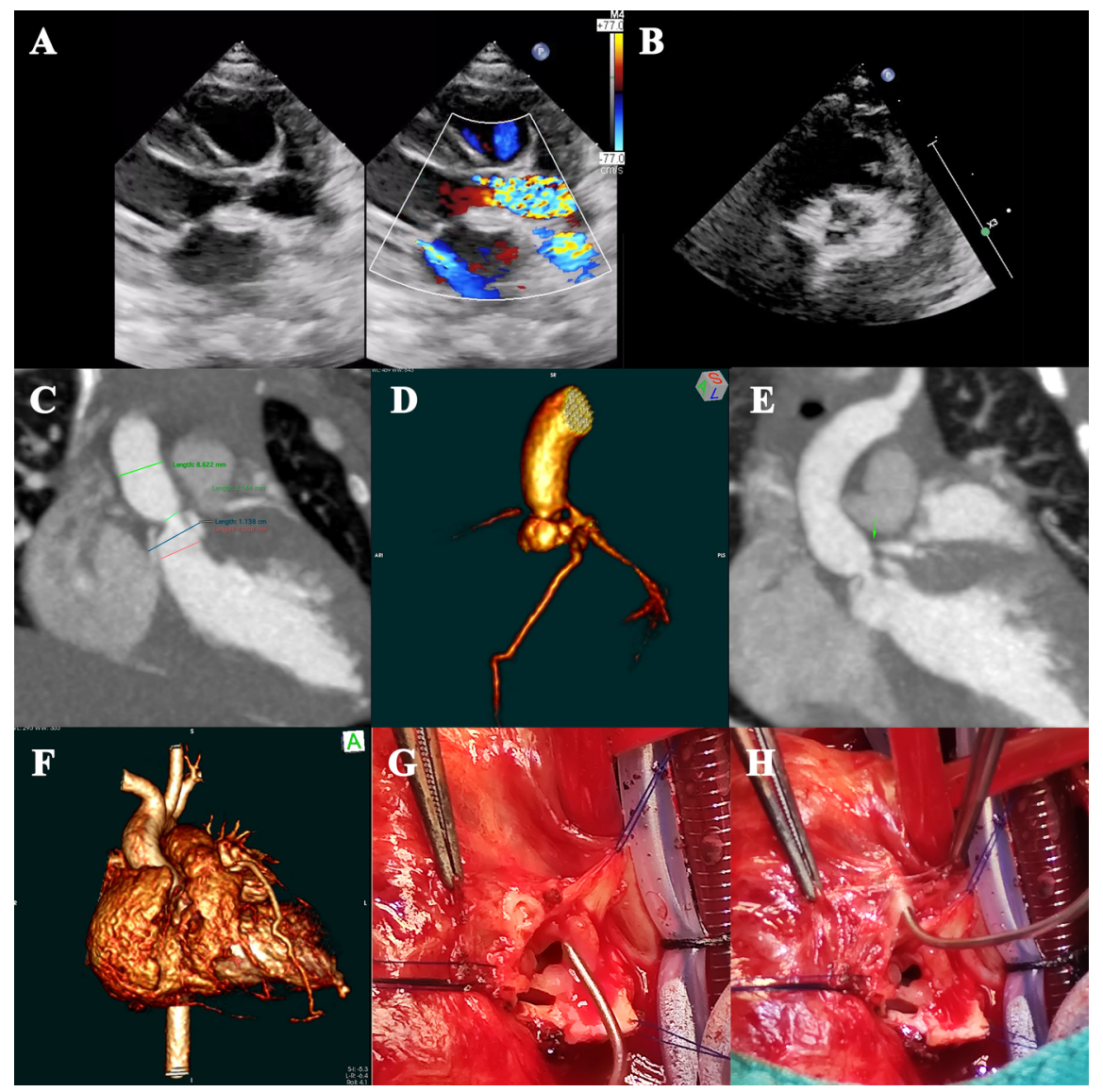

